\title{
Multifunctional biomedical applications of magnetic nanodiamond
}

Elena Perevedentseva

Artashes Karmenyan

Yu-Chung Lin

Chang-You Song

Zhe-Rui Lin

Ashek-I-Ahmed

Chia-Chi Chang

Svetlana Norina

Valentina Bessalova

Nikolai Perov

Olga Levinson

Boris Zousman

Chia-Liang Cheng 


\title{
Multifunctional biomedical applications of magnetic nanodiamond
}

\author{
Elena Perevedentseva, ${ }^{\mathrm{a}, \mathrm{b}}$ Artashes Karmenyan,, ${ }^{\mathrm{a}}$ Yu-Chung Lin,, ${ }^{\mathrm{a}, \mathrm{c}}$ Chang-You Song, ${ }^{\mathrm{a}}$ Zhe-Rui Lin, ${ }^{\mathrm{a}}$ \\ Ashek-I-Ahmed, ${ }^{\mathrm{a}}$ Chia-Chi Chang, ${ }^{\mathrm{a}}$ Svetlana Norina, ${ }^{\mathrm{d}}$ Valentina Bessalova, ${ }^{\mathrm{d}}$ Nikolai Perov, ${ }^{\mathrm{d}}$ \\ Olga Levinson,e Boris Zousman, ${ }^{e}$ and Chia-Liang Cheng ${ }^{\mathrm{a}, *}$ \\ aNational Dong Hwa University, Department of Physics, Hualien, Taiwan \\ ${ }^{b}$ P. N. Lebedev Physics Institute of Russian Academy of Sciences, Moscow, Russia \\ 'Institute of Physics, Academia Sinica, Taipei, Taiwan \\ dMoscow State University, Department of Physics, Moscow, Russia \\ ${ }^{\mathrm{e}}$ Ray Techniques, Jerusalem, Israel
}

\begin{abstract}
The research and development for biomedical applications are recently focused on multifunctional nanoparticles. To integrate various functionalities, different methods of modifying the particle's physical properties are developed. Among the considered, nanodiamond (ND) is a promising candidate for the development of multifunctional complex due to its variable features in size, structure, surface chemistry, physical properties, and biocompatibility. In addition to its well-studied structural, surface, electrochemical and photonic properties, strong magnetism of ND can be observed. In the present work, magnetically modified ND is introduced in terms of its bioapplications. Along with the soft ferromagnetism of ND, the increased fluorescence at oneand two-photon excitation is realized. Utilizing the combined magnetic and fluorescence properties of the magnetically modified ND, fluorescence imaging, fluorescence lifetime imaging and manipulation of cells by magnetic field are demonstrated. The perspectives to use the magnetic ND for drug delivery, cells magnetic separation and filtration, in bioengineering to control the cell distribution combined with imaging and treatment are discussed. (C) 2018 Society of Photo-Optical Instrumentation Engineers (SPIE) [DOI: 10.1117/1.JBO.23.9.091404]
\end{abstract}

Keywords: nanodiamond; magnetic nanoparticles; magnetic nanodiamond; theranostics; bioimaging; controllable drug delivery. Paper 170786SSR received Dec. 9, 2017; accepted for publication Feb. 22, 2018; published online Mar. 21, 2018; corrected Apr. 16, 2018.

\section{Introduction}

The recent intense developed nanoparticle (NP) applications in biology and medicine are essentially determined by their multifunctional uses. Particularly, for example, theranostic application $^{1-3}$ usually includes combining functionalities of drug delivery or other medical treatments with multimodal imaging. Therefore, the particles that are able to combine the desired physical/chemical properties attract close attention. Among all NPs, nanodiamond (ND) appears to be a promising candidate for the multifunctional applications ${ }^{2}$ due to its surface, electrochemical, and optical properties; and the availability in various size and surface structure, and, most importantly, the demonstrated biocompatibility. ${ }^{3}$ In spite of some limitations, a number of facilities for ND theranostic applications have been demonstrated. These include stimulus-responsible ND drug delivery and release ${ }^{2}$ multimodal imaging utilizing luminescence properties of $\mathrm{ND}$, allowing fluorescence imaging at one- and two-photon excitation, ${ }^{2,3}$ fluorescence lifetime imaging (FLIM) ${ }^{4}$ Raman spectroscopic analysis and mapping; ${ }^{3}$ and optical sensing on the base of energy transfer ${ }^{5}$ and magnetic sensing utilizing spin properties of structural defects ${ }^{6}$. One possibility to get multifunctional nanoparticles is to modify the nanoparticle to enhance the desired properties for certain application, for example, methods to enhance ND fluorescence in different spectral ranges. ${ }^{2,5,7}$

*Address all correspondence to: Chia-Liang Cheng, E-mail: clcheng@gms. ndhu.edu.tw
In this work, we discuss magnetically modified ND and its biomedical applications. Magnetic ND (MND) can combine properties and advantages of the ND and magnetic nanoparticle. The most developed and clear way to prepare magnetic ND is doping with magnetic atoms. ${ }^{8}$ However, in some cases, such ND can show some level of cytotoxicity and be not biocompatible.

Here we report, the magnetic ND was synthesized and treated without the use of metals and metal oxides using the method proposed by ray techniques. ${ }^{9}$ The origin of its magnetism is unclear. As for magnetic properties of diamond in general, pure diamond structure combines diamagnetic and paramagnetic components, ${ }^{10}$ but in an intermediate graphite-diamond structure the conditions for spin ordering and magnetic interactions can be created. ${ }^{10}$ Such structure can be realized in ND. Ferromagnetic properties of carbon nanostructures are discussed for theoretically predicted structures and are found experimentally; the methods to obtain such properties without embedding magnetic atoms are developed using mechanical, electromagnetic treatments, ${ }^{11}$ hydrogenation, ${ }^{12,13}$ creation defects and doping (with nonmagnetic atoms), ${ }^{13}$ and surface state modification. ${ }^{14}$ Additionally, spin properties of structural defects of ND are studied ${ }^{15}$ and the applications of ND are discussed, such as magnetic resonance imaging (MRI) agent for MRI ${ }^{16}$ and for quantum sensing. ${ }^{6,15}$

Bioapplications of magnetic nanoparticles have also developed into a topical issue. The magnetic nanoparticles of various composition, size, shape, and surface properties are developed

$1083-3668 / 2018 / \$ 25.00$ @ 2018 SPIE 
for different purposes. For instance, they can serve as probes in living tissue and be controlled noninvasively from the outside by an external magnetic field, wherein the magnetic field action on the diamagnetic biotissues is negligible. ${ }^{17}$ The most developed applications of magnetic nanoparticles are used as a MRI marker at local magnetic hyperthermia and its applications; controlled drug delivery, guided by magnetic field; cell separation and filtration. ${ }^{18}$ With the aforementioned possibilities, in the present work, the magnetic-modified ND is characterized, analyzed, and demonstrated from the point of view of bioapplications.

\section{Methods and Materials}

\subsection{Magnetic Fluorescent Nanodiamond}

The ND, called further RayND, was synthesized by laser treatment of specially prepared hydrocarbon targets in liquid medium without the use of metals or metal oxides. ${ }^{19}$ These NDs produced by controlled technology of light hydrodynamic pulse (LHDP) ${ }^{9}$ have average size of $4.3 \mathrm{~nm}$ and are rather similar to ND obtained by detonation synthesis (DND), just purer and more uniform due to the use of metal-free raw materials and the controlled method of the synthesis. After isolation from hydrocarbon blend, synthesized RayND particles underwent a two-step chemical-thermal modification using metal-free chemicals. First, RayND was treated thermally for ordering carbon radicals on the ND surface resulting in the unusual ferromagnetism of unknown nature. Then to provide the colloidal stability, to enhance the ND dispersion in aqueous solutions and increase in the sedimentation stability, of diamond nanofluids, the obtained magnetic ND powder was further chemically modified in metal-free solvents, nitrogenized, which for unclear reason changed its optical properties (e.g., PL). Note, that the process of RayND surface modification is not disclosed in this paper. Magnetically and optically modified ND (RayND-M) were analyzed and compared with DND received with nominal average size of crystallites 3 to $10 \mathrm{~nm}$ (Microdiamant AG, Switzerland).

\subsection{RayND-M Characterization}

The diamond structure of ND (DND, RayND, and RayND-M) was confirmed by x-ray diffraction analysis (XRD) using D8 Advance diffractometer (Bruker AXS, Karlsruhe, Germany), performed as described in Ref. 19. The obtained XRD patterns are identical and indicate that all samples have almost the same crystalline structure close to $100 \%$ diamond with the average size of primary particles automatically defined by Scherrer's formula of 4.3 to $4.5 \mathrm{~nm}$.

Magnetic properties of the RayND-M, and the comparison with DND, were measured using LakeShore 7407 vibrating sample magnetometer (VSM, Lake Shore Cryotronics) at ambient conditions. The ND samples (about $15 \mathrm{mg}$ ) were packed in the plastic diamagnetic bag with a size of $5 \times 5 \mathrm{~mm}$ and a thickness of $0.5 \mathrm{~mm}$. The free bag was also investigated for magnetic moment field dependence. The sample magnetic moment was determined as a difference of the sample and free bag signals at the same magnetic field.

The composition of the investigated samples was estimated via energy-dispersive X-ray analysis (EDX), with scanning electron microscopy imaging using FE-SEM JEOL JSM 7000F (JEOL, Japan) to check the presence of magnetic atoms in the RayND-M.
For EDX-SEM analysis as well as for measurements of Raman, FTIR, fluorescence spectra, and fluorescence lifetime measurements, ND water suspension was dropped on the $\mathrm{Si}$ substrate $(1 \mathrm{~cm} \times 1 \mathrm{~cm})$ and dried in a desiccator at room temperature.

The particles (and their aggregates) size distribution as well as $\zeta$-potential in water were measured using the dynamic light scattering (DLS) method in a Zetasizer nano ZS (Malvern Instruments, United Kingdom), with a 4-mW, 633-nm wavelength $\mathrm{He}-\mathrm{Ne}$ laser, detection angle $173 \mathrm{deg}$ at $\mathrm{pH} \sim 6.8$.

To estimate predominant structure of crystallites, Raman spectra were measured using a Renishaw 1000B (Renishaw, United Kingdom) spectrometer equipped with a CW laser (DPGL-2100F; Photop Suwtech, China) with 532-nm wavelength; and a confocal Raman $\alpha-300 \mathrm{~S}$ (Witec, Germany) with a 488-nm wavelength argon laser (Melles Griot). Surface functional groups were analyzed using a Fourier transform infrared spectroscopy (Bomem MB154 FTIR spectrometer, ABB Bomem, Switzerland) in situ using a mercury cadmium telluride liquid nitrogen cooled detector under vacuum with chamber base pressure $\sim 3 \times 10^{-6}$ Torr.

To study photonic properties of the RayND-M, absorption spectra were measured for ND water suspensions using a UV-visible JASCO V-550 spectrometer (JASCO, Japan) at room temperature. For the fluorescence spectra, the Renishaw 1000B and $\alpha$-300S confocal Raman spectrometers were used. Analysis of the RayND-M fluorescence lifetime at two-photon excitation and FLIM at their interaction with target biological systems were performed using a system on the base of a femtosecond tunable Ti-Sapphire laser Chameleon Ultra-II (Coherent), the used excitation wavelength was in the 760- to 800-nm range, repetition rate $80 \mathrm{MHz}$, pulse duration $140 \mathrm{fs}$, and laser power at about $3 \mathrm{~mW}$. For FLIM, a 2-D scanner (EINST Technology, Singapore) was used. The registration was recorded in the 450- to 650-nm spectral range with the single photon counting system PicoHarp 300 (PicoQuant, Germany) and cooled PMT connected with an Olympus IX 71 optical microscope, $40 \times$ objective.

\subsection{RayND-M Interaction with Cells}

The biointeraction of RayND-M was studied using baby hamster kidney cells (BHK) and human lung alveolar carcinoma cells (A549) cell cultures obtained from Bioresource Collection and Research Center, Taiwan (BCRC, Taiwan). The A549 cells were cultured in RPMI1640 medium (Gibco, Invitrogen, United Kingdom), BHK cells were cultured in Dulbecco's modified eagle medium (DMEM) (Gibco, Invitrogen, United Kingdom), all are supplemented with 2-mM L-glutamine (Invitrogen), $1.5 \mathrm{~g} / \mathrm{L}$ sodium bicarbonate (Sigma), and $10 \%$ fetal bovine serum (FBS) (Gibco/Life Technologies). Cells were maintained under standard cell culture conditions in an incubator (Galaxy 170S, Eppendorf) at $95 \%$ air and $5 \% \mathrm{CO}_{2}$ and $37^{\circ} \mathrm{C}$. Culture medium was replaced with fresh medium every 48 or $72 \mathrm{~h}$. Cells were detached by treatment with $0.5 \%$ trypsin and $2.6 \mathrm{mM}$ ethyl-enediaminetetraacetic acid (Gibco/Life Technologies); cultures were subcultured routinely at $\sim 80 \%$ confluence.

Human serum albumin (HSA, Sigma, Ronkonkoma) was physically adsorbed on RayND-M. The albumin solution with a concentration of $40 \mathrm{mg} / \mathrm{ml}$ and an aqueous suspension of the ND with concentration of $4 \mathrm{mg} / \mathrm{ml}$ were mixed in a $1: 1$ ratio. After thorough mixing for $2 h$, the mixture was centrifuged 
to separate ND from the protein adsorbed on the surface. The separated sediment was washed twice in distilled water. This method provides the formation of a stable ND-protein complex..$^{20}$ To confirm the adsorption, the albumin content in the solutions was determined using a UV-Visible spectrometer JASCO V-550 before its mixing with ND and after the isolation of ND-protein complexes.

To study the interaction with the BHK cells, the cells were treated with $20 \mu \mathrm{g} / \mathrm{ml}$ of RayND-M added to the cell growth medium for $4 \mathrm{~h}$, whereas nonreacted ND has been washed out. Then the cells were fixed with $3.7 \%$ formaldehyde (FA) on the coverslips. The cells with ND were investigated with a confocal microscope and FLIM, to visualize the ND penetration into the cell. For investigations with the confocal microscope, cell cytosol was dyed with Alexa Fluor 674 (Sigma); the nuclei were stained with Hoechst33342 (Sigma). A TCS SP5 Laser Scanning Fluorescence Confocal Microscope (Leica, Germany) was used. To define the ND location in the cell structures, the scan along vertical direction ( $Z$-scan) was performed. For ND intercellular distribution visualization, the RayND-M fluorescence was excited by $488 \mathrm{~nm}$ and collected in the range from 560 to $600 \mathrm{~nm}$; Hoechst fluorescence was excited with $405 \mathrm{~nm}$ and detected at 450 to $490 \mathrm{~nm}$; cytoplasm stained with Alexa Fluor 647 was excited with $633 \mathrm{~nm}$ with detection at 640 to $690 \mathrm{~nm}$.

For FLIM investigation, the cells were not dyed. The FLIM investigation at two-photon excitation was performed as described above.

The cytotoxicity was estimated by MTT (3-(4,5-dimethylthiazol-2-yl)-2,5-diphenyltetrazolium bromide) assay ${ }^{21}$ using NDHSA complex and BHK cell line. BHK cells were seeded in quantity of $4 \times 10^{5}$ cells/well in a 96-well microtiter plate in DMEM supplemented with 2-mM L-glutamine (Invitrogen) and $10 \%$ of $\mathrm{FBS}$ and incubated at $37^{\circ} \mathrm{C}$ and $5 \% \mathrm{CO}_{2}$ for $24 \mathrm{~h}$. After incubation, the medium was removed. Then the cells were treated with a fresh medium containing ND-HSA of different RayND-M concentrations (5 to $200 \mu \mathrm{g} / \mathrm{ml}$ ) for 24 and $48 \mathrm{~h}$. After this procedure, the medium was removed and the cells were treated for $4 \mathrm{~h}$ with MTT reagent (with the concentration of $2.5 \mathrm{mg} / \mathrm{ml}$ ). The surviving cells converted MTT-agent to formazan, a blue-purple color when dissolved in dimethyl sulfoxide (DMSO). The solution was removed and $200 \mu \mathrm{l}$ of DMSO was added to dissolve the formazan. The optical absorption of the treated cells and control (nontreated) cells was measured at $570 \mathrm{~nm}$ (OD 570) with ELISA reader (MRX revelation Microplate Reader, DYNEX). Relative percentages of surviving cells were calculated by dividing the absorbance of the treated cells with that of the control measured in each experiment.

For the experiment with magnetic guidance of the cells containing RayND-M by a magnetic field, the cells were detached from the bottom of the culture dish, suspended in the cellular medium, and investigated with microscopy and FLIM.

\subsection{Utilizing RayND-M Magnetic Properties}

\subsubsection{Magnetic field guidance of RayND-M/cell complexes}

The possibility of magnetic guidance of ND and ND-containing cells was demonstrated for particles in water and for RayND-Mcontaining living cells. To create a nonuniform magnetic field, a thin steel needle (with diameter of $0.18 \mathrm{~mm}$ ) was magnetized with neodymium magnet $(\mathrm{NdFeB}$, disk of diameter of $5 \mathrm{~mm}$;
National Imports). For a preliminary testing, RayND-M was suspended in the distilled water with a concentration of $20 \mu \mathrm{g} / \mathrm{ml}$. To demonstrate a magnetic guidance and control by the magnetic field of the spatial distribution of the NDcontaining cells, the cells were detached from the bottom of the culture dish and suspended in the cellular medium. The guidance of particles and their aggregates and the cells with embedded RayND-M by the magnetic field were observed with a conventional microscope (Olympus IX 71, objective with magnification $40 \times$ ) and with FLIM.

\subsubsection{Magnetic field-controlled RayND-M/cell complex distribution and positioning}

To demonstrate the possibility to control the spatial distribution of the cells during culturing by the magnetic field, suspension of RayND-M-containing cells was mixed with the cells without the ND and recultured together for 12 to $36 \mathrm{~h}$ in the presence of the magnetic field. The magnetic field was applied locally to the selected area of the cultural dish, using the permanent magnets. After removing the magnets, the areas treated and nontreated with the magnetic field were analyzed with a confocal microscope and FLIM to see the cells distribution.

\section{Results and Discussion}

\subsection{Characterization of the Nanodiamond}

In Fig. 1(a), the magnetization curve of RayND-M is shown and compared with the magnetization curve of diamagnetic DND. For the RayND-M, the strong magnetization with magnetic hysteresis was observed revealing soft ferromagnetism of this ND. In comparison with nanomagnetite particles, the magnetization of RayND-M is almost 100 times less, so the interaction (and aggregation also) of the particles caused by magnetic dipoledipole interaction should be weaker and this fact could be considered as the advantage. Figure 1(b) shows the elemental analysis of the RayND-M; the sample's SEM image is shown in Fig. 1(c). Characteristic EDX spectrum of RayND-M demonstrates a very low content of magnetic atoms ( $\mathrm{Fe}, \mathrm{Co}$, and Ni). Numerically estimated for several measurements the highest content was observed for $\mathrm{Fe}$, being from 0 to $0.2 \mathrm{wt}$. $\%$. Note that DND powders from diverse suppliers usually have incombustible impurities of 0.5 to 3.2 wt. \% with $\mathrm{Fe}$ content defined as 0.10 to $0.55 \mathrm{wt} \% \%^{22}$ however, they do not exhibit tangible ferromagnetic properties. Therefore the origin of ferromagnetism in RayND-M could be considered due to their surface structural modification rather than the elemental contents. However, the mechanism is still unclear.

The magnetic properties of carbon are recently discussed in some theoretical and experimental works. ${ }^{10}$ The models that in principle can be realized in ND are suggested. For example, it has been shown that if some carbon $\mathrm{sp}^{2}$-hybridized atoms in a graphite two-dimensional network are substituted by three-valence atoms such as $\mathrm{sp}^{3}$-hybridized carbon atoms, the resulting magnetic moment can be high. The system becomes nonplanar as the tetrahedral-coordinated carbon atoms link to atoms of the graphite-like layers. Thus, a stable all-carbon structure with the ferromagnetic interaction can be created. However, the magnetism nature of nanocarbon is still unclear and is not fully understood.

The possibilities and methods to create carbon and nanocarbon structures with strong magnetic properties are discussed 

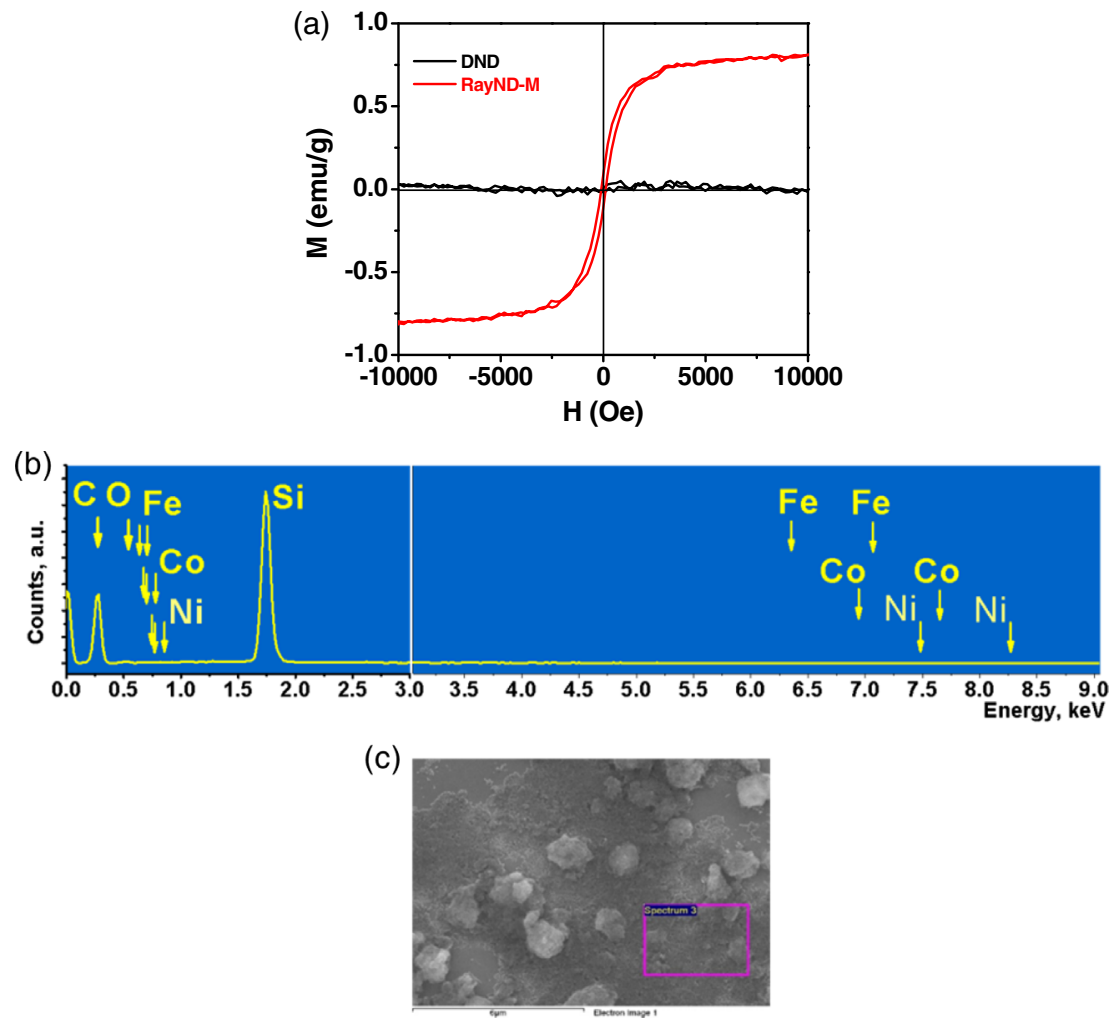

Fig. 1 Magnetic properties of RayND-M. (a) Magnetization curve of RayND-M (I) [in comparison with ordinary DND (II)]. (b) Elemental analysis (EDX spectrum) of RayND-M. Negligible (no) containing of magnetic atoms is observed. (c) SEM image of the RayND-M powder.

recently, especially imparting paramagnetism and ferromagnetism to ND significantly increases the ND attractiveness for its use in biomedical researches and applications. ${ }^{10,11}$

Figure 1(c) shows large aggregates of the studied nanoparticles in the state of dry powder. The DLS analysis of the nanoparticles in the water suspension showed an average size of $90 \pm 10 \mathrm{~nm}$. These results also correspond to the particles aggregation. In contrast to the magnetization curve, the Raman spectrum of the RayND-M [Fig. 2(a)] is similar in principle with characteristic spectra of fine ND (DND). It reveals a peak of $\mathrm{sp}^{3}$-hybridized carbon, confirming the diamond structure of the particles. The peak is broadened and shifted, relative to the peak of bulk diamond at $1332 \mathrm{~cm}^{-1}$, to 1322 to $1325 \mathrm{~cm}^{-1}$. This shift is attributed to the phonon confinement effect at decreasing the crystallites size to 3 to $10 \mathrm{~nm} .^{23}$ The shift of the diamond band to lower wavenumbers at the decrease of crystallite size is often accompanied by shifting the characteristic band for $\mathrm{sp}^{2}$-bonded carbon or G-band from $1590 \mathrm{~cm}^{-1}$ to higher wavenumbers $\left(1630 \mathrm{~cm}^{-1}\right.$ in presented case). It is ascribed as a superposition of G-band with a vibration of some surface groups ${ }^{24}$ or to interstitial defect, which consists of an isolated $\mathrm{sp}^{2}$-bonded carbon pair in the position of one carbon atom in a diamond lattice. ${ }^{25}$ Note that the cross section of Raman scattering of diamond is low, so relatively intense diamond and D-band reveals significant diamond $\left(\mathrm{sp}^{3}\right)$ fraction.

The FTIR spectrum [Fig. 2(b)] of RayND-M shows the zsurface carboxyl group characterized by resolving the $\mathrm{C}=\mathrm{O}$ stretching in the 1720 to $1780 \mathrm{~cm}^{-1}$ range and $\mathrm{O}-\mathrm{H}$ bending 1630 to $1640 \mathrm{~cm}^{-1}$. The lower frequency range from 1000 to $1300 \mathrm{~cm}^{-1}$ is usually assigned for the $\mathrm{C}-\mathrm{O}$ functional group in ether/ester structure present on RayND-M. A corresponding
$\mathrm{OH}$ stretching feature is observed at 3000 to $3600 \mathrm{~cm}^{-1}$, which could be related to the carboxyl group and water adsorption. Atmospheric $\mathrm{CO}_{2}$ is in wavenumber 2300 to $2350 \mathrm{~cm}^{-1}$ and $\mathrm{C}-\mathrm{H}$ related to hydrocarbon or surface adsorbed hydrogen on $\mathrm{sp}^{3}$-dangling carbon is found in the range of 2800 to $3000 \mathrm{~cm}^{-1}$. As compared with RayND-M, there is no substantial difference with nonmodified DND. The $\zeta$-potential was measured in bidistilled water suspension to be $-32.8 \mathrm{mV}$ at $\mathrm{pH}$ 6.8, which is consistent with carboxylated ND. Therefore, the crystal structure of RayND-M, observed with Raman, and surface properties characterized with FTIR are similar to ND and RayND; ${ }^{3,26}$ this suggests some well-developed methods can be used to attach molecules of interest to the RayND-M surface for drug delivery, targeted imaging, etc.

In contrast to most NDs, the photonic properties of RayND$\mathrm{M}$ would be quite promising for multifunctional applications. It was found well detectable at confocal imaging at excitation in UV-visible range and two-photon excitation also gives a strong signal, which allow lifetime analysis and FLIM.

Figure 3(a) shows the UV-visible absorption and photoluminescence spectra measured using 488- and 532-nm wavelengths CW lasers excitations. Lifetime decay curve and FLIM of RayND-M powder at two-photon excitation using a 790-nm wavelength femtosecond laser, pulse duration $140 \mathrm{fs}$, and repetition rate $80 \mathrm{MHz}$ are shown in Fig. 3(b). Fluorescence lifetime is short and estimated to be $\sim 0.2$ to $0.5 \mathrm{~ns}$, being close to instrument response function (IRF). The signal is intense and the lifetime differs significantly from lifetimes of the most endogenous fluorophores, so RayND-M is well detectable both at twophoton excitation of fluorescence lifetime and at one-photon fluorescence measurements. Note that the origin of enhanced 

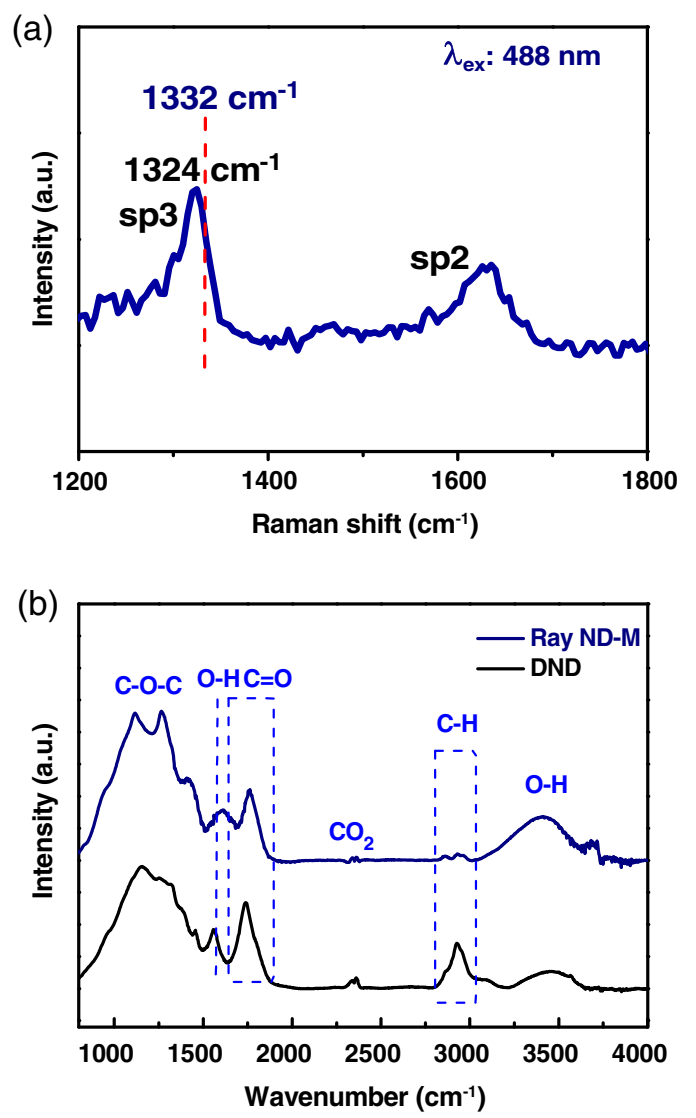

Fig. 2 (a) Raman spectra and (b) FTIR spectra of RayND-M in comparison with DND.
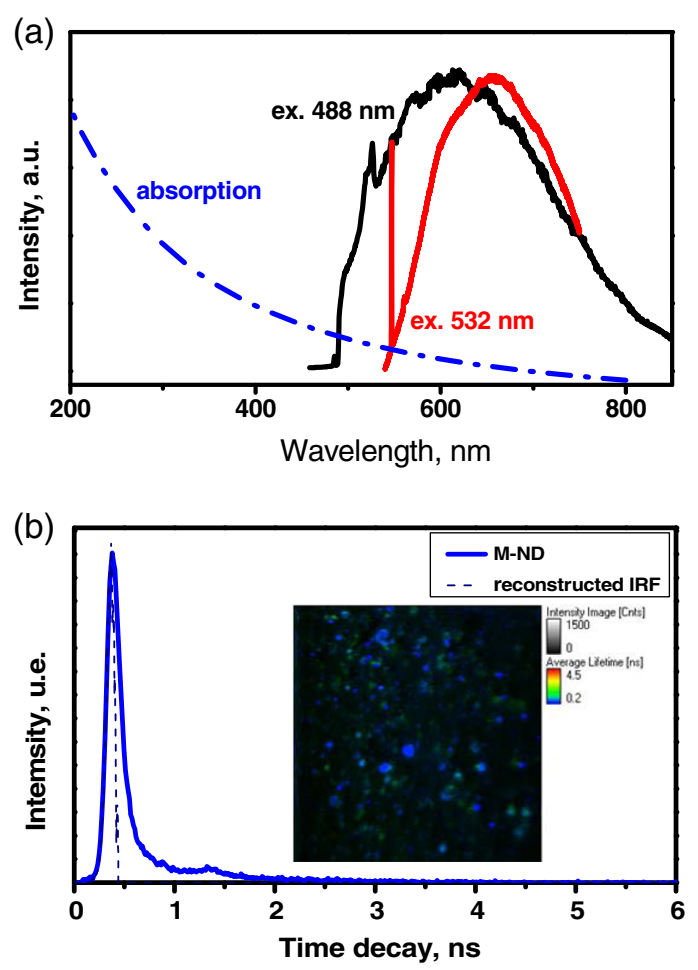

Fig. 3 Fluorescence properties of RayND-M. (a) Absorption and fluorescence spectra at one-photon excitation; (b) lifetime decay and FLIM (inset) of RayND-M powder at two-photon excitation with femtosecond laser at $790-\mathrm{nm}$ wavelength. fluorescence emission from magnetic-modified ND is also not clear yet and can be discussed together with the origin of magnetic properties, but it is subject to other consideration.

\subsection{Interaction with Cell}

RayND-M penetration into the cell is demonstrated using confocal microscopic imaging. In Fig. 4(a), the confocal fluorescence image of the BHK cell is presented after the cell incubation with RayND-M for $4 \mathrm{~h}$, with the concentration of ND in the culture medium (DMEM) set at $20 \mu \mathrm{g} / \mathrm{ml}$. In the image, ND is shown in green, the cell cytoplasm in red, and nuclei is shown in blue. Yellow reveals the ND entered the cytoplasm. The cross-sectional $z$-scan images on one cell at changing positions in the $z$-axis with $1-\mu \mathrm{m}$ step were performed for observation of the ND inside the cell. It clearly reveals ND engulfment by the cell and their localization in the cytoplasm, like other ND, the penetration of which into cells of various types was observed and the clathrin-dependent endocytosis was studied and confirmed. ${ }^{27}$

Fluorescence lifetime analysis [Fig. 4(b)] and imaging [Figs. 4(c) and 4(d)] at two-photon excitation also allow visualizing ND interaction with the cell. Since the RayND-M fluorescence lifetime is much shorter than that of the cell autofluorescence, RayND-M is well observed in FLIM, which enables to define the ND location inside the cell, to monitor, and to quantify the metabolic state of the living cell and its viability can be used as lifetime analyzing, for example, endogenous fluorophores, such as NADH and FAD. ${ }^{28}$

Low cytotoxicity of the RayND-M was observed using the MTT assay with the viability of the BHK cell line (Fig. 5). No concentration dependence or time dependence (in the times and concentrations range used) are observed. The encapsulation of RayND-M with protein albumin (HSA) can additionally reduce the low cytotoxicity of RayND-M to a negligible level. Albumin adsorption shown previously as a factor increasing the ND biocompatibility, not only provides the safe interaction with a biological target, but also helps to control the ND deaggregation and dispersibility. ${ }^{20}$ In addition, the ND encapsulation with albumin enables controlling the desired interaction with surfaceattached drugs, which is highly important for the development of new reliable nanoparticles-based drug delivery systems. ${ }^{29}$

With these observations, magnetic ND was shown very promising for bioapplications due to its low toxicity and to fluorescence properties that provide possibilities for multimodal imaging combining excitations in very wide range, including nearIR, and different methods of signal detection. Note that observed fluorescence properties of RayND-M are significantly more convenient for bioimaging as compared with other nonmodified NDs (such as DND). And additional new facilities for the RayND-M multifunctional use are opened due to the significant magnetism of this ND. In the following, we consider some options and demonstrate with preliminary experiments and discuss the possibilities of the applications development.

\subsection{RayND-M Magnetic Properties Applications}

The possibility of the RayND-M guided with magnetic field was demonstrated using a nonuniform magnetic field from a magnetized steel needle of diameter $0.18 \mathrm{~mm}$, magnetized with a strong permanent magnet. The RayND-M particles were suspended in the bidistilled water. When the needle was immersed in the suspension, the ferromagnetic particles were attracted to 
(a)
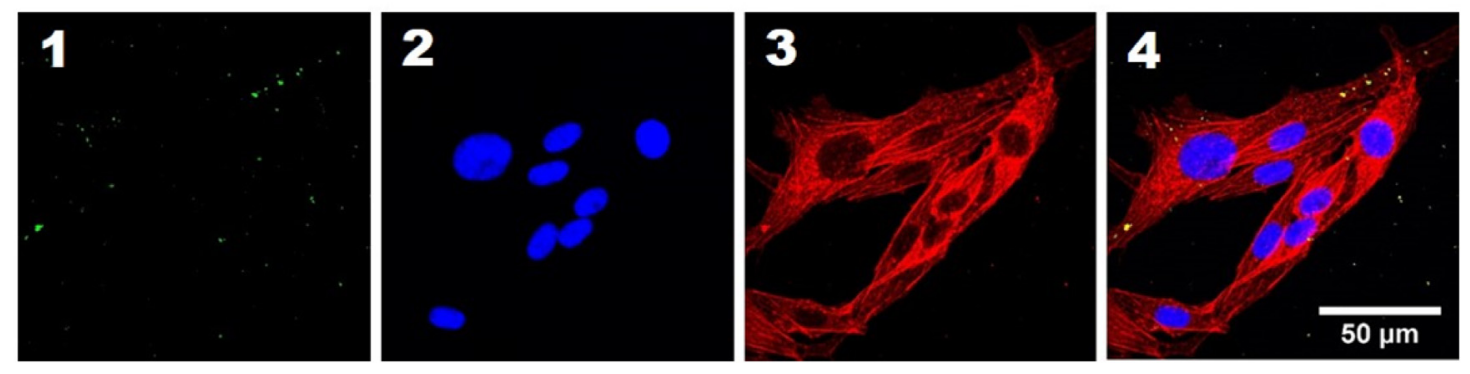

(b)

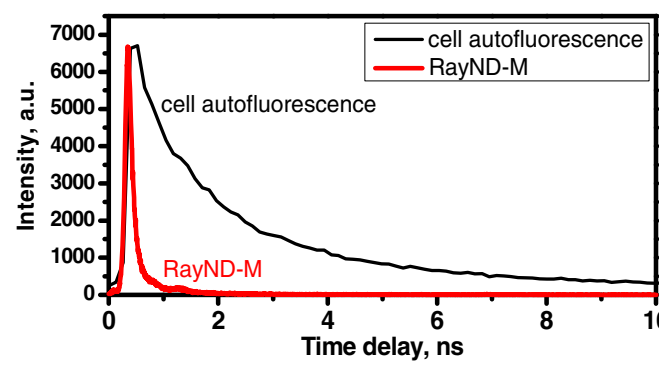

(c)

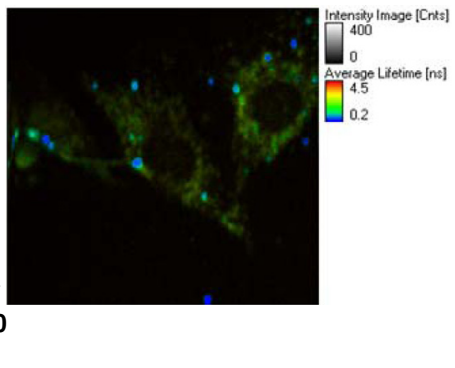

(d)

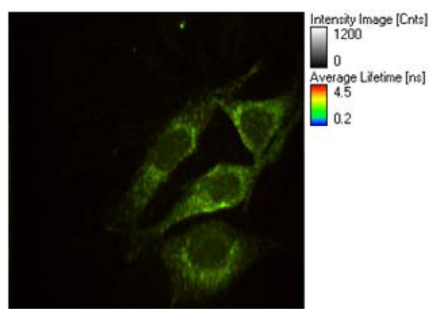

Fig. 4 Imaging of RayND-M interaction with cell. Observation RayND-M in BHK cell, concentration of RayND-M in the medium is $20 \mu \mathrm{g} / \mathrm{ml}$, cocultured time $4 \mathrm{~h}$. (a) Laser confocal fluorescence image: (1) RayND-M fluorescence is excited with $488 \mathrm{~nm}$ and detected in the 530- to 690-nm range, shown in green; (2) the cell cytoplasm was dyed with Fluor 674; (3) cell nuclei with Hoechst33342; and (4) merging of 1 to 3 with RayND-M shown in yellow. (b) Lifetime decays of cell autofluorescence and fluorescence of RayND-M in BHK cell; (c) FLIM of the cell with RayND-M; and (d) FLIM of control cell without RayND-M.
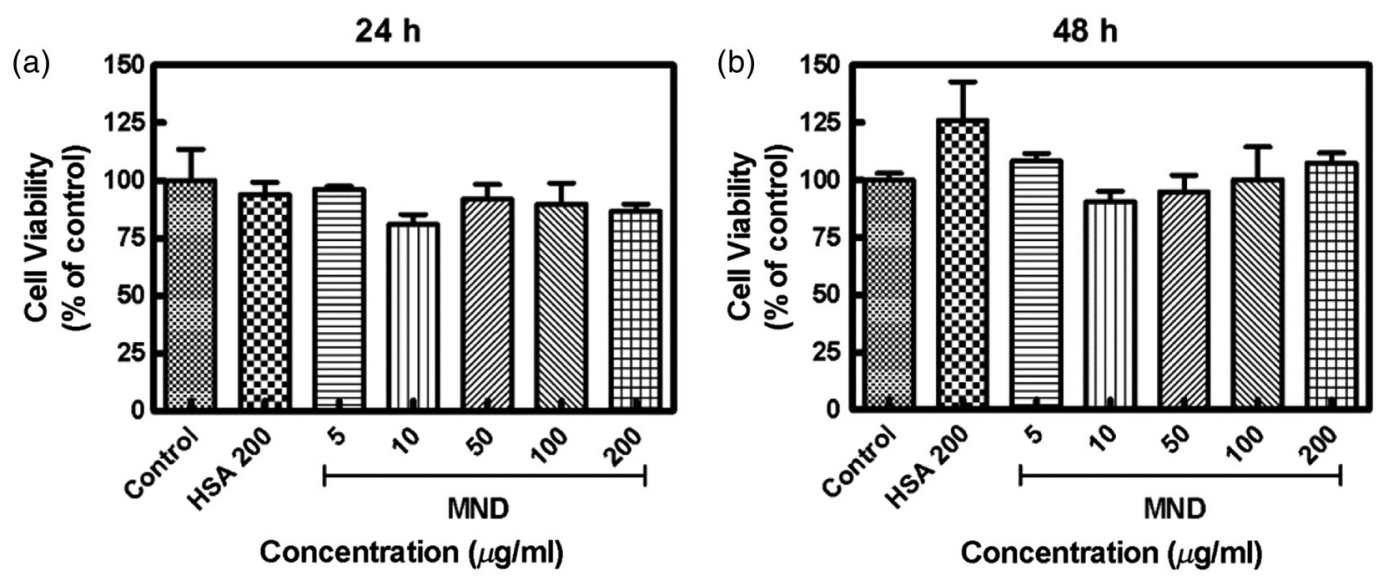

Fig. 5 RayND-M cytotoxicity on the BHK cells estimated via MTT test at various concentrations of RayND-M in the complex with HSA after (a) $24 \mathrm{~h}$ and (b) $48 \mathrm{~h}$ of the ND treatment.

the region of the magnetic field gradient as a result of magnetophoresis. ${ }^{30,31}$ Figure 6(a) shows the scheme of this simple experiment. The moving of particles and their aggregates in the needle's magnetic field were observed with an optical microscope and with lifetime imaging, respectively. The optical image in Fig. 6(b-1) was obtained at the beginning of the experiment; Fig. 6(b-2) is the FLIM of the particles attracted to the needle; and Fig. 6(b-3) shows the microscopic image of the needle with attracted particles just after FLIM measurement. This series of images show that particles in water or in a medium can be moved by magnetic field and in principle can be used for developing methods of drug delivery.

The motion of the water- or medium-suspended particles driven by a magnetic field can be used in controlled dispersion of therapeutic agents within liquid- and gel-filled cavities of an organism. Nonspecific delivery and distribution in such a system are driven by diffusion and are limited by the molecule (or NP carrier) concentration and size, surrounding medium properties, such as viscosity. Application of magnetic NP controlled by the external magnetic field provides the possibility to optimize 
(a)

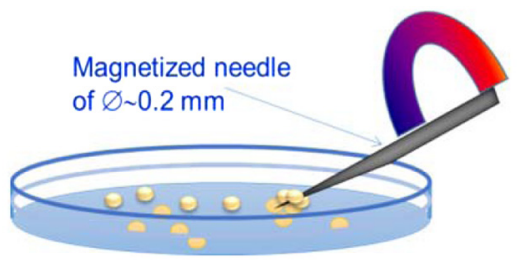

(b)

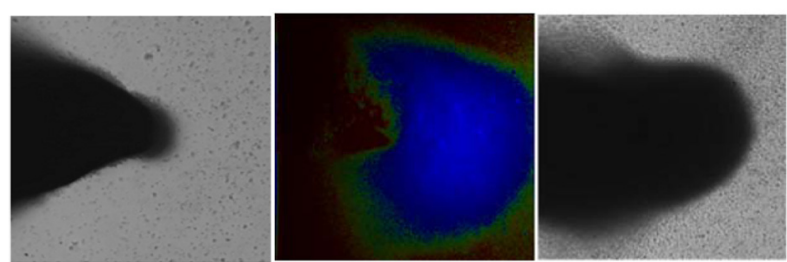

(c)

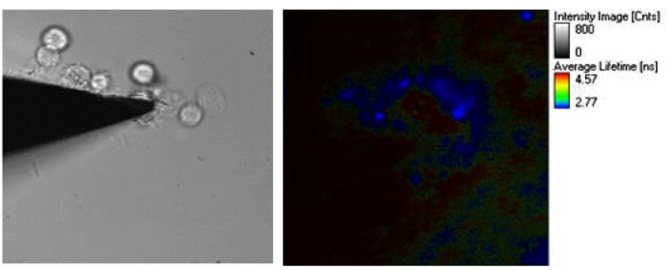

Fig. 6 Magnetic guidance. (a) Schematic of the guidance of magnetic particles in the magnetic field. (b) Microscopic images of the needle in the RayND-M water suspension: left, the experiment beginning; center, FLIM of the particles, attracted to the needle; right, microscopic image of the needle with attracted particles after FLIM measurement. (c) RayND-M labeled A549 cells attracted to the magnetized needle: left, microscopic image and right, FLIM.

the controlled delivery. ${ }^{32}$ This scheme [Fig. 6(a)] can also be used to demonstrate the possibility of the magnetic field's guided cells labeled with RayND-M.

Magnetic labeling of cells provides new possibilities for the remote cells manipulations for various purposes, as well as for investigations including MRI and treatments, such as a local magnetic hyperthermia. The methods of cells labeling with magnetic nanoparticles are developed; ${ }^{30}$ among these particles, ND with superbiocompatibility and well-studied pathways of cells penetration also become a very promising candidate when being magnetically modified. The magnetically labeled cells manipulations with magnetic field and cells magnetic separation ${ }^{30,32}$ have been realized in applications such as detection of rare cancer cells in circulating blood, detection of fetal cells in maternal blood, and modification of cellular content of the graft for cellular therapies. ${ }^{30,33}$

To validate this idea, A549 cells was incubated with RayND$\mathrm{M}$ for $4 \mathrm{~h}$, than detached from the cultivation dish bottom to be suspended in the medium (PBS). Their moving in the magnetic field created by the magnetized needle was observed. The effect of the applied magnetic field on these cells was observed with microscopic imaging and FLIM. The cell attraction evidenced from the cells moving toward the needle is shown in Fig. 6(c). Importantly, the attracted cells [Fig. 6(c)-left] contain RayNDM, as the FLIM shows [Fig. 6(c)-right].

Using magnetic nanoparticles can also be considered in the context of bioengineering. It should be noted that the combining of bioengineering and magnetic fabrication for the purpose of graft engineering was first tried about 30 years ago ${ }^{30}$ and references therein. Recently it is continuing to be developed, so the problem of the nanoparticles optimization remains important to overcome some obstacles and limitations. On the other hand, among the NP, the use of ND in bioengineering has been presented. For example, NDs are considered for bone engineering due to their mechanical properties and biocompatibility. ${ }^{34}$ Thus, it is sensible to discuss using RayND-M for bioengineering combined with magnetic nanofabrication, MND allows also controlling and visualization of the tissue forming.

For simple estimation of the MND applicability in this capacity, monolayer cell culturing was used. To show the possibility of MND used for the control of cell distribution during the growth of cell culture, the mixture of MND-labeled and nonlabeled A549 cells was recultured in the presence of the local magnetic field. The magnetic field from the neodymium permanent magnet was applied to selected area of the culturing dish with the labeled and nonlabeled cells mixture in a cellular medium. The cells were cultivated together for 12 to $36 \mathrm{~h}$. Then the cell culture was observed with confocal microscopy and FLIM to see how the cells with and without MND distribute in the presence of the local magnetic field. The schematic of the experiment and result is shown in Fig. 7. Both confocal
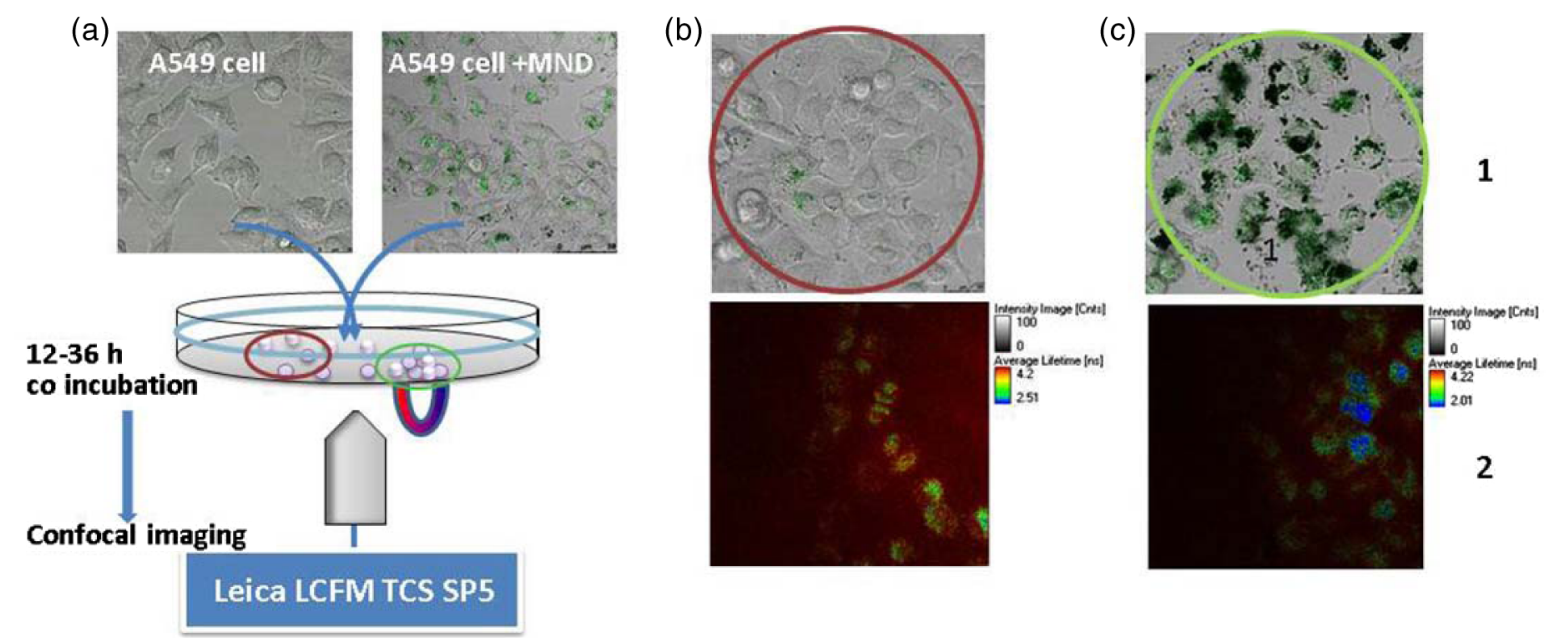

Fig. 7 Magnetic field-controlled RayND-M/cell complex distribution and positioning. (a) Schematic of the experiment and imaging of growing cells preincubated with RayND-M in the presence of local magnetic field: distribution of the cell out of (b) and in (c) MF area; (1) confocal image and (2) FLIM image. 
fluorescence imaging and FLIM show cells with MND localized close to the magnet. Note also that the distribution of RayNDM-labeled cells in the magnetic field could be controlled by the field geometric configuration and parameters of cell cultivation (ND concentration, cocultivation time). This demonstrates the principle to use the magnetic ND to control the cell distribution combined with multimodal imaging; and opens new prospects for its applications in bioengineering allowing simultaneous controlled growth of the tissue, tracking of particles during the process and visualization of obtained structures.

\section{Conclusion}

In the present work, magnetically modified ND is introduced in terms of its multifunctional bioapplications. Fluorescent ferromagnetic metal-free ND powder (RayND-M) has been obtained by surface modification of ND produced by the LHDP method (the process of the surface modification is not disclosed in this paper). The diamond structure and purity of ND were defined by EDX, Raman, SEM, and the surface was characterized with FTIR; soft ferromagnetism of the ND was detected by a vibrating sample magnetometer. Together with the ferromagnetism of the nanoparticles, the increased fluorescence at one- and twophoton excitation is demonstrated. Note, however, that the origins of the ferromagnetism as well as of enhancement of fluorescence emission in the result of magnetic modification of the ND are still unclear and can be considered together.

High biocompatibility of RayND-M was confirmed by MTT analysis using the BHK cell line. RayND-M uptakes and its localization in the cytoplasm similar to other NDs have been observed. The possibility of magnetic guidance of ND in cells and magnetic manipulation of ND containing cells in tissue has been demonstrated by the example of A549 cells cocultured with RayND-M. Strong magnetism, fluorescence, and noncytotoxicity of the modified magnetic ND enables significant breakthroughs in controlled bioengineering, tissues regeneration, early diagnostics, and targeted drug delivery.

\section{Disclosures}

The authors have no relevant financial interests in this article and no potential conflicts of interest to disclose.

\section{Acknowledgments}

The authors appreciate the financial support of this research by the Ministry of Science and Technology (MoST) of Taiwan, Grant No. MOST 106-2112-M-259-009-MY3 and by the European Commission REA Grant No. 690945 CARTHER.

\section{References}

1. D. E. Lee et al., "Multifunctional nanoparticles for multimodal imaging and theragnosis," Chem. Soc. Rev. 41(7), 2656-2672 (2012).

2. V. Vaijayanthimala et al., "Nanodiamond-mediated drug delivery and imaging: challenges and opportunities," Expert Opin. Drug Delivery 12(5), 735-749 (2015).

3. E. Perevedentseva et al., "Biomedical applications of nanodiamond in imaging and therapy," Future Med. Nanomed. 8(12), 2041-2060 (2013).

4. W. W.-W. Hsiao et al., "Fluorescent nanodiamond: a versatile tool for long-term cell tracking, super-resolution imaging, and nanoscale temperature sensing," Acc. Chem. Res. 49(3), 400-407 (2016).

5. R. Fudala et al., "FRET enhanced fluorescent nanodiamonds," Curr. Pharm. Biotechnol. 14(13), 1127-1133 (2014).

6. Y. Wu et al., "Diamond quantum devices in biology," Angew. Chem. Int. Ed. 55(23), 6586-6598 (2016).
7. I. I. Vlasov et al., "Molecular-sized fluorescent nanodiamonds," Nat. Nanotechnol. 9, 54-58 (2014).

8. C. H. Chen et al., "Fe doped magnetic nanodiamonds made by ion implantation," Sci. Rep. 7, 41938 (2017).

9. B. Zousman and O. Levinson, "Pure nanodiamonds produced by laserassisted technique," Chapter 5 in Nanodiamond, O. Williams, Ed., pp. 221-252, RSC Nanoscience and Nanotechnology, London (2014).

10. T. Makarova, "Magnetic properties of carbon structures," Semiconductors 38, 615-638 (2004).

11. V. E. Orel et al., "Magnetic characteristics and anticancer activity of a nanocomplex consisting of detonation nanodiamond and doxorubicin," J. Superhard Mater. 34, 179-185 (2012).

12. G. Zhang et al., "Superconducting ferromagnetic nanodiamond," ACS Nano 11, 5358-5366 (2017).

13. K. Kenmochi et al., "Materials design of ferromagnetic diamond," Jpn. J. Appl. Phys. 44(2), L51 (2005).

14. N. I. Kiselev et al., "Electrical and magnetic properties of nanodiamond and pyrocarbon composites," Russ. J. Gen. Chem. 83(11), 2173-2181 (2013).

15. M. Geiselmann et al., "Three-dimensional optical manipulations of a single electron spin," Nat. Nanotechnol. 8, 175-179 (2013).

16. D. E. J. Waddington et al., "Nanodiamond-enhanced MRI via in situ hyperpolarization," Nat. Commun. 8, 15118 (2017).

17. S. Odenbach, Colloidal Magnetic Fluids: Basics, Development and Application of Ferrofluids, Springer, Berlin-Heidelberg (2009).

18. L. Mohammed et al., "Magnetic nanoparticles for environmental and biomedical applications: a review," Particuology 30, 1-14 (2017).

19. E. Perevedentseva et al., "Nanodiamonds of laser synthesis for biomedical applications," J. Nanosci. Nanotechnol. 15(2), 1045-1052 (2015).

20. L.-W. Tsai et al., "Nanodiamonds for medical applications: interaction with blood in vitro and in vivo," Int. J. Mol. Sci. 17(7), 1111 (2016).

21. T. L. Riss et al., "Cell viability assays," in Assay Guidance Manual, G. S. Sittampalam et al., Eds., Eli Lilly \& Company and the National Center for Advancing Translational Sciences, Bethesda, Maryland (2004).https://www.ncbi.nlm.nih.gov/books/NBK144065/

22. R. Yu et al., "Determination of impurities in detonation nanodiamonds by gamma activation analysis method," Diamond Relat. Mater. 55, 7786 (2015).

23. S. Osswald et al., "Phonon confinement effects in the Raman spectrum of nanodiamond," Phys. Rev. B 80, 075419 (2009).

24. V. Mochalin, S. Osswald, and Y. Gogotsi, "Contribution of functional groups to the Raman spectrum of nanodiamond powders," Chem. Mater. 21, 273-279 (2009).

25. J. O. Orwa et al., "Raman investigation of damage caused by deep ion implantation in diamond," Phys. Rev. B 62(9), 5461-5472 (2000).

26. M. V. Baidakova et al., "Structure of nanodiamonds prepared by laser synthesis," Phys. Solid State 55(8), 1747-1753 (2013).

27. K.-J. Huang et al., "Phagocytosis and immune response studies of macrophage-nanodiamond interactions in vitro and in vivo," J. Biophotonics 10, 1315-1326 (2017).

28. T. S. Blacker et al., "Separating NADH and NADPH fluorescence in live cells and tissues using FLIM," Nat. Commun. 5, 3936 (2014).

29. J. Mariam, S. Sivakami, and P. M. Dongre, "Albumin corona on nanoparticles-a strategic approach in drug delivery," Drug Delivery 23(8), 2668-2676 (2016).

30. J.-K. Lim et al., "Magnetophoresis of nanoparticles," ACS Nano 5(1), 217-226 (2011).

31. Y. Jing et al., "Quantitative intracellular magnetic nanoparticle uptake measured by live cell magnetophoresis," FASEB J. 22(12), 4239-4247 (2008).

32. M. Zborowski and J. J. Chalmers, Magnetic Cell Separation, Vol. 32, Elsevier Science, Laboratory Techniques in Biochemistry and Molecular Biology, Amsterdam, The Netherlands (2008).

33. S. J. Kuhn, D. E. Hallahan, and T. D. Giorgio, "Characterization of superparamagnetic nanoparticle interactions with extracellular matrix in an in vitro system," Ann. Biomed. Eng. 34(1), 51-58 (2006).

34. X. Wu et al., "Functionalization of bone implants with nanodiamond particles and angiopoietin-1 to improve vascularization and bone regeneration," J. Mater. Chem. B 5, 6629-6636 (2017).

Elena Perevedentseva received her PhD in physics from Moscow State University, Moscow, Russia. She is working at the National 
Dong Hwa University, Taiwan. Her research is focused on microscopic and spectroscopic studies of nanoparticle interaction with biological objects. She also collaborates as a senior researcher with the P. N. Lebedev Physics Institute of the Russian Academy of Sciences, Moscow, Russia, working in the field of optics and spectroscopy.

Artashes Karmenyan received his $\mathrm{PhD}$ in physics from Yerevan State University (YSU), Armenia. He was head of the Spectroscopy Laboratory in "Laserayin Technika" Institute, YSU, then joined the Laboratory of Laser Methods for Diagnosis and Therapy, Cancer Research Center, Moscow, Russia. Since 2001 he is working in National Yang-Ming University and National DongHwa University, Taiwan, in the fields of laser spectroscopy (Raman, fluorescence, time-resolved), biomedical applications, and noninvasive optical techniques for mammalian embryos research.

Yu-Chung Lin received his BSc degree from the Department of Biomechatronic Engineering, National Chiayi University, Taiwan, in 2009. He graduated from the National Dong Hwa University with a master's degree in physics in 2011 and received his $\mathrm{PhD}$ in June 2016 in physics. His research is in the field of Raman spectroscopy and fluorescence spectroscopy applications in life science.

Chang-You Song received his BSc degree from the Department of Physics, Notional Dong Hwa University, Taiwan, in 2016. While he was an undergraduate, he did his undergraduate research focused on the cellular models of the magnetic nanoparticle interaction with various cell lines of interested.

Zhe-Rui Lin received his master's degree from NDHU in Hualien, Taiwan. He studied at the nanocarrier for the therapy of cancer cell which was via modulation the nanoparticle to enhance the drug efficiency. Furthermore, he is the most interested in the fluorescence spectroscopy applications in biosystems.

Ashek-I-Ahmed has achieved his BSc and MSc degrees in physics from the Department of Physics, Rajshai University, Bangladesh. Currently, he is pursing $\mathrm{PhD}$ on applied physics under supervision of Professor Chia-Liang Cheng (Charlie) at the Department of
Physics, National Dong Hwa University, Taiwan. His research topic is "Surface reaction dynamics of nanodiamond and its bioapplications". His research interest is on the field of optical spectroscopy, biomechanics, and nanophysics.

Chia-Chi Chang is an undergraduate student at Department of Physics of National Don Hwa University, Hualien, Taiwan. He is interested in using Raman and fluorescence spectroscopy to analyze nanodiamond as a drug carrier for biomedical applications.

Svetlana Norina achieved her PhD in physics from Moscow State University in 1983. She is doing her research in Magnetism Chair of Physics Department of Moscow State University focusing on problems of biomagnetism.

Valentina Bessalova has graduated from Lomonosov MSU in 2014 and works as the junior researcher of the Magnetism Department of Lomonosov MSU, research interests are in investigation of nanoparticles and nanocomposites.

Nikolai Perov is a professor in Lomonosov Moscow State University. Does his research in the Magnetism Chair of Physics Department since 1977, has visiting experience-Tohoku University, Toyohashi University of Technology, National University of Singapore, DuisburgEssen University. Nowadays he is the head of the Magnetism Department of Lomonosov MSU, research interests are in investigation of magnetic properties of smart and intelligent materials.

Chia-Liang Cheng is a specialist in Raman and infrared spectroscopy. He received his PhD from Department of Physics, University of Oregon, USA, in 1993, and did postdoctoral research at the University of California, Berkeley, and at the Institute of Atomic and Molecular Sciences, Academia Sinica, Taiwan. Currently, he is a professor of physics at the Physics Department, National Dong Hwa University, Taiwan. His interest is in carbon nanostructural materials, especially nanodiamond, and their biomedical applications.

Biographies for the other authors are not available. 